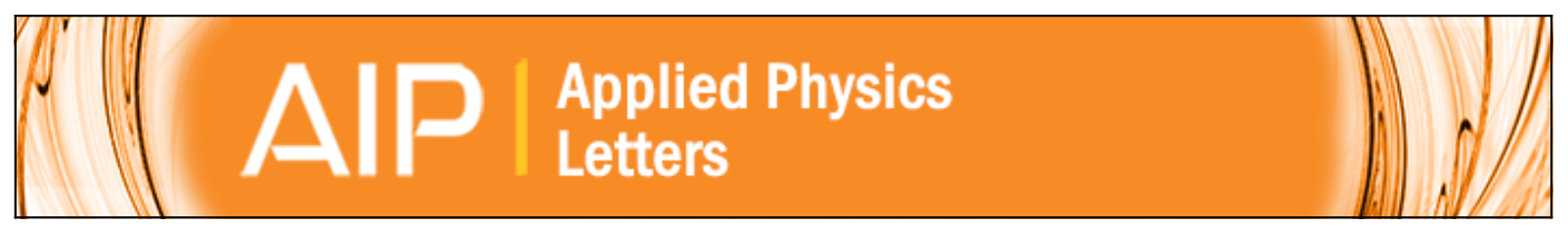

\title{
Excess noise in GaAs and AIGaAs avalanche photodiodes with GaSb absorption regions-composite structures grown using interfacial misfit arrays
}

A. P. Craig, C. J. Reyner, A. R. J. Marshall, and D. L. Huffaker

Citation: Applied Physics Letters 104, 213502 (2014); doi: 10.1063/1.4879848

View online: http://dx.doi.org/10.1063/1.4879848

View Table of Contents: http://scitation.aip.org/content/aip/journal/apl/104/21?ver=pdfcov

Published by the AIP Publishing

\section{Articles you may be interested in}

Time resolved gain and excess noise properties of InGaAs/InAIAs avalanche photodiodes with cascaded discrete gain layer multiplication regions

J. Appl. Phys. 113, 093705 (2013); 10.1063/1.4794345

Origin of dark counts in In $0.53 \mathrm{Ga} 0.47 \mathrm{As} / \mathrm{In} 0.52 \mathrm{Al} 0.48 \mathrm{As}$ avalanche photodiodes operated in Geiger mode Appl. Phys. Lett. 86, 063505 (2005); 10.1063/1.1861498

$1.31 \mu \mathrm{m}$ GaAsSb resonant-cavity-enhanced separate absorption, charge and multiplication avalanche photodiodes with low noise

J. Appl. Phys. 93, 774 (2003); 10.1063/1.1526933

Temperature investigation of dark current and its electrical noise in GaAs/AIGaAs multiquantum well photodiodes J. Appl. Phys. 85, 1211 (1999); 10.1063/1.369343

Characteristics of GaAs and AIGaAs homojunction avalanche photodiodes with thin multiplication regions Appl. Phys. Lett. 71, 3883 (1997); 10.1063/1.120533

Frustrated by

old technology?

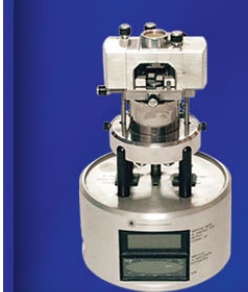

Is your AFM dead

and can't be repaired?

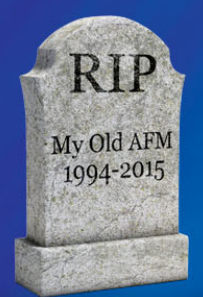

Sick of bad customer support?

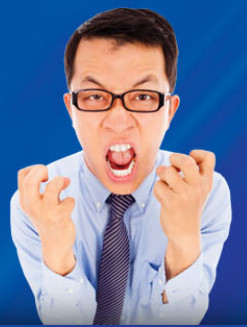

It is time to upgrade your AFM

Minimum $\$ 20,000$ trade-in discount for purchases before August 31st

Asylum Research is today's technology leader in AFM 


\title{
Excess noise in GaAs and AIGaAs avalanche photodiodes with GaSb absorption regions-composite structures grown using interfacial misfit arrays
}

\author{
A. P. Craig, ${ }^{1}$ C. J. Reyner ${ }^{2}$ A. R. J. Marshall, ${ }^{1}$ and D. L. Huffaker ${ }^{2,3}$ \\ ${ }^{1}$ Physics Department, Lancaster University, Lancaster LAI 4YB, United Kingdom \\ ${ }^{2}$ Department of Electrical Engineering, UCLA, Los Angeles, California 90095, USA \\ ${ }^{3}$ California NanoSystems Institute, UCLA, Los Angeles, California 90095, USA
}

(Received 26 March 2014; accepted 14 May 2014; published online 28 May 2014)

\begin{abstract}
Interfacial misfit arrays were embedded within two avalanche photodiode (APD) structures. This allowed GaSb absorption layers to be combined with wide-bandgap multiplication regions, consisting of GaAs and $\mathrm{Al}_{0.8} \mathrm{Ga}_{0.2} \mathrm{As}$, respectively. The GaAs APD represents the simplest case. The $\mathrm{Al}_{0.8} \mathrm{Ga}_{0.2} \mathrm{As}$ APD shows reduced dark currents of $5.07 \mu \mathrm{Acm}^{-2}$ at $90 \%$ of the breakdown voltage, and values for effective $k=\beta / \alpha$ below 0.2 . Random-path-length modeled excess noise is compared with experimental data, for both samples. The designs could be developed further, allowing operation to be extended to longer wavelengths, using other established absorber materials which are lattice matched to GaSb. (C) 2014 Author(s). All article content, except where otherwise noted, is licensed under a Creative Commons Attribution 3.0 Unported License. [http://dx.doi.org/10.1063/1.4879848]
\end{abstract}

Avalanche photodiodes (APDs) are known to have enhanced sensitivities compared with simple $p-i-n$ photodiodes. At the same time, improved detectors for the short and mid-infrared spectral ranges, between 1.4 and $8 \mu \mathrm{m}$, are increasingly sought after for various applications, including telecommunications, military hardware, ${ }^{1}$ gas sensing, ${ }^{2}$ and night-vision equipment. Long-wavelength APDs could be suitable for these purposes, especially where low photon fluxes are present. However, further development is required to combine longer-wavelength operation with higher sensitivities and lower dark currents and noise. Designs based on $\mathrm{GaSb}$ and InAs substrates are an attractive choice, since a range of narrow-gap layers can then be grown latticematched. These layers include binary GaSb and InAs, as well as ternaries (e.g., InAsSb), quaternaries (e.g., InGaAsSb) and strained layer superlattices. Indeed, InAs APDs, which have a cut-off wavelength of $3.5 \mu \mathrm{m}$, were recently shown to have very low avalanche noise. ${ }^{3}$ However, they were also reported to suffer from significant surfaceleakage currents and a potential for unwanted band-to-band tunneling effects. One alternative approach is to use a strained-layer-superlattice for the multiplication region. This has been demonstrated on both $\mathrm{GaSb}$ and $\mathrm{InP}$ substrates, with reported cut-off wavelengths of $4.92 \mu \mathrm{m}^{4}$ and $2.5 \mu \mathrm{m},{ }^{5}$ respectively. Elsewhere, lattice matched InGaAsSb/ $\mathrm{AlGaAsSb}$ separate-absorption-and-multiplication (SAM) structures have been developed. These have typically been grown on GaSb substrates, with cut-off wavelengths around $2.2 \mu \mathrm{m}$ being reported. ${ }^{6,7}$ By taking advantage of both the absorption properties of a narrow-gap material and the multiplication properties of a wide-gap material, SAM-APD designs can offer an amalgam solution where longwavelength devices with high sensitivities are required. An extension of this approach is pursued in the present work.

The interfacial misfit (IMF) array is a technique for molecular beam epitaxial (MBE) growth, allowing high-quality, relaxed epilayers to be deposited on lattice-mismatched substrates without the need for a metamorphic buffer. ${ }^{8,9}$ In this process, the strain is relieved within a few monolayers of the interface by a self-ordered network of $90^{\circ}$ misfit dislocations, leaving intact the bulk properties of the deposited crystal. For the growth of GaSb onto GaAs in particular, derivative devices including light emitting diodes (LEDs) ${ }^{10}$ and lasers ${ }^{11}$ have already been demonstrated. In addition, recent publications include a report of GaInAsSb photodiodes, operating in the $2-2.4 \mu \mathrm{m}$ wavelength range. ${ }^{12}$ These were reported to have a level of performance comparable with similar detectors grown on native GaSb substrates. In this work, IMF arrays were used to directly combine GaAs and AlGaAs multiplication layers (lattice constant $5.65 \AA$ ) with GaSb absorption layers (lattice constant 6.09 $\AA$ ). The GaAs design represents the simplest case and a proof-of-principle. This is developed in the AlGaAs design to realize lower excess noise, a thinner structure and further suppression of the depletion currents. In both devices, a narrow-bandgap GaSb absorber material gives photocurrent at wavelengths up to $1.7 \mu \mathrm{m}$. It is anticipated that other absorber materials-specifically, those which are lattice-matched to $\mathrm{GaSb}$ - could also be used, allowing longer wavelength operation. This would be possible by using the IMF GaSb layer as a thin buffer.

The two structures, which are depicted in Figure 1, were grown using a Veeco Gen930 MBE reactor. In each case, oxide desorption from the GaAs substrate was performed first, at $600^{\circ} \mathrm{C}$. The substrate temperature was subsequently reduced to $580{ }^{\circ} \mathrm{C}$ for the growth of the $n^{+}$-GaAs cladding regions. For the GaAs design, a $p^{-}$multiplication region was grown next. In the AlGaAs design, this is replaced with a shorter, unintentionally doped multiplication region and a $p^{+}$ charge sheet. The charge sheet strongly confines the high electric field within the multiplication region. Note that, for the GaAs design, the charge associated with the IMF array 


\begin{tabular}{|c|c|}
\hline \multirow{3}{*}{ $\pm 111 \pm$ IMF array } & \\
\hline & $50 \mathrm{~nm}$ p-GaSb $1.0 \times 10^{19} \mathrm{~cm}^{-3}$ \\
\hline & $250 \mathrm{~nm}$ p-GaSb $1.0 \times 10^{18} \mathrm{~cm}^{-3}$ \\
\hline & $500 \mathrm{~nm}$ p-GaSb $6.0 \times 10^{16} \mathrm{~cm}^{-3}$ \\
\hline $50 \mathrm{~nm}$ p-GaSb $1.0 \times 10^{19} \mathrm{~cm}^{-3}$ & $40 \mathrm{~nm}$ GaAs buffer laver (undoped) \\
\hline $250 \mathrm{~nm}$ p-GaSb $6.0 \times 10^{16} \mathrm{~cm}^{-3}$ & $65 \mathrm{~nm} p-\mathrm{Al}_{0.8} \mathrm{Ga}_{0.2} \mathrm{As} 1.0 \times 10^{18} \mathrm{~cm}^{-3}$ \\
\hline $4.2 \mathrm{~nm}$ GaAs buffer (undoped) & $50 \mathrm{~nm} \mathrm{Al}{ }_{0.8} \mathrm{Ga}_{0.2} \mathrm{As}$ (undoped) \\
\hline $525 \mathrm{~nm}$ p-GaAs $2.5 \times 10^{16} \mathrm{~cm}^{-3}$ & $200 \mathrm{~nm} n-\mathrm{Al}_{0.8} \mathrm{Ga}_{0.2} \mathrm{As} 2.0 \times 10^{18} \mathrm{~cm}^{-3}$ \\
\hline $600 \mathrm{~nm}$ n-GaAs $2.0 \times 10^{18} \mathrm{~cm}^{-3}$ & $600 \mathrm{~nm} n-G a A s 2.0 \times 10^{18} \mathrm{~cm}^{-3}$ \\
\hline GaAs substrate (semi-insulating) & GaAs substrate (semi-insulating) \\
\hline GaAs Design & AlGaAs Design \\
\hline
\end{tabular}

FIG. 1. Shows the cross-sectional schematic of the two structures. Left: GaAs design, Right: AlGaAs design.

itself is sufficient to prevent the field spreading into the GaSb absorber. ${ }^{13}$ Growth then proceeded as follows for both samples. First, a short, undoped GaAs buffer was deposited. Next, after a brief interrupt with no applied As flux, which leaves the growth-surface $\mathrm{Ga}$ terminated, an $\mathrm{Sb}$ flux was applied in order to initiate the IMF interface. At the same time, the temperature was reduced to $510^{\circ} \mathrm{C}$. Growth of the $\mathrm{GaSb}$ absorption and cap layers followed. The V-III ratios were approximately 10 for GaAs and AlGaAs growth and 4.2 for $\mathrm{GaSb}$ growth. All growth rates were between $0.2-1.0$ $\mathrm{MLs}^{-1}$. The $n$ - and $p$-type dopants were $\mathrm{Si}$ and $\mathrm{Be}$, respectively. Annular contacts were evaporated using Ti/Pt/Au for $p$-GaSb and AuGe/Ni/Au for $n$-GaAs. Circular mesas were defined using an inductively coupled plasma (ICP) reactive ion etch (RIE) and $\mathrm{BCl}_{3} / \mathrm{Ar}$ etch chemistry.

Figure 2 shows detailed current-voltage (IV) data, collected using a Keithley 2400 SourceMeter. Illumination was provided by under-filling the device using a $15 \mathrm{~mW} 1.55 \mu \mathrm{m}$ laser, incident via a cleaved, single-mode optical fiber. For both samples, the dark currents below breakdown were noted to be greatly reduced compared with levels expected for a homojunction GaSb $p-i-n$ diode. ${ }^{14}$ This is attributed to the confinement of the electric field within the multiplication (wide-bandgap) regions, and remains true even after the onset of $1.55 \mu \mathrm{m}$ photocurrent. In turn, it is inferred that photogenerated carriers are able to travel from the GaSb absorption layers by diffusion, crossing the IMF interfaces. With $90 \%$ of breakdown voltage applied, current densities of $560 \mu \mathrm{Acm}^{-2}$ and $5.07 \mu \mathrm{Acm}^{-2}$ were noted, for the GaAs design and AlGaAs design, respectively. Conspicuous band-to-band tunneling effects were noted to be absent; these might have been expected if high fields were present in the $\mathrm{GaSb}$ regions. Furthermore, surface leakage currents, typically around $1 \times 10^{-5} \mathrm{Acm}^{-2}$ at $-5.0 \mathrm{~V}$ for $200 \mu \mathrm{m} \mathrm{GaSb}$ $p-i-n$ diodes, grown and processed by the authors using the

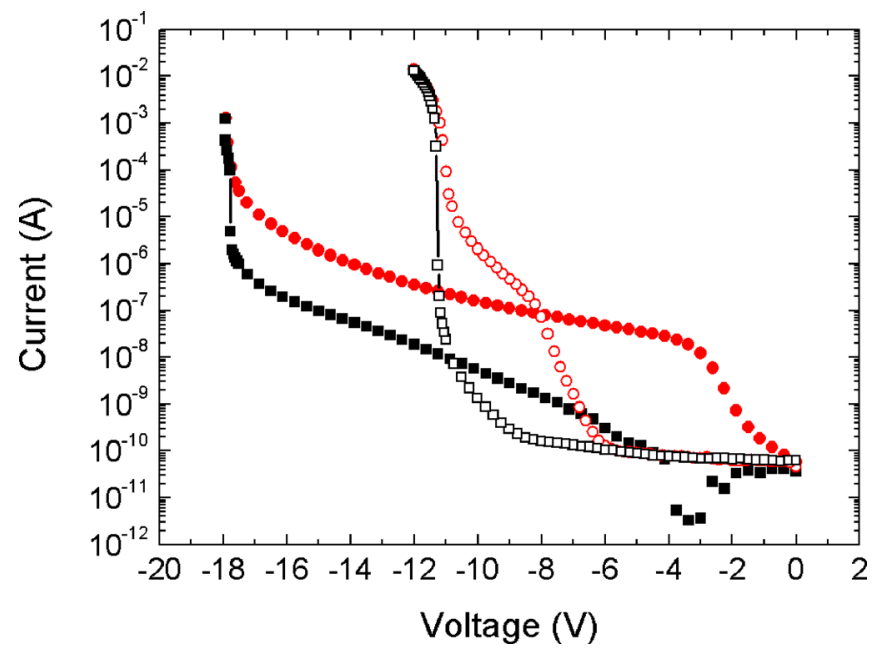

FIG. 2. Shows IV data for $200 \mu \mathrm{m}$ diameter mesas. Dark currents: GaAs design ( $\square$ ), AlGaAs design ( $\square$ ). Current under illumination from $1.55 \mu \mathrm{m}$ laser: GaAs design (O), AlGaAs design $(\bigcirc)$. The solid lines are guides to the eye.

same procedures as the samples in the present work, were noted to have been suppressed. This is attributed to the wide-bandgap layers acting to increase the shunt resistance associated with the surface states. The multiplication was noted to be independent mesa area for both samples, showing that edge breakdown effects are absent and, thus, do not influence the excess noise results presented here. Figure 3 shows spectral response curves for both samples, taken at $300 \mathrm{~K}$ using a Stanford SR830 lock-in amplifier in combination with a Bentham TMc300 monochromator. By extrapolation of the squared response, plotted against energy, cut-off wavelengths of around $1.70 \mu \mathrm{m}$ and around $1.75 \mu \mathrm{m}$ were calculated, for the GaAs design and the AlGaAs design, respectively. It should be noted that the cut-off wavelength is slightly increased in the AlGaAs design, owing to the thicker absorption region, which leads to a greater probability of absorption for longer-wavelength photons.

Extensive capacitance-voltage (CV) measurements were further taken for both samples, as illustrated in Figure 4.

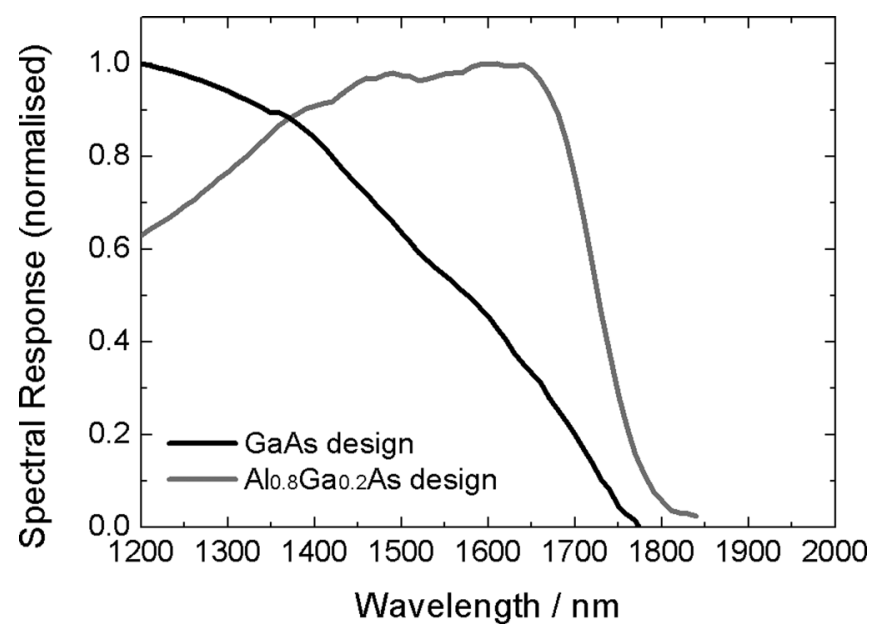

FIG. 3. Shows $300 \mathrm{~K}$ spectral response curves, for both samples. The bias voltages were $17.3 \mathrm{~V}$ and $8.9 \mathrm{~V}$, for the GaAs design and the AlGaAs design, respectively. 


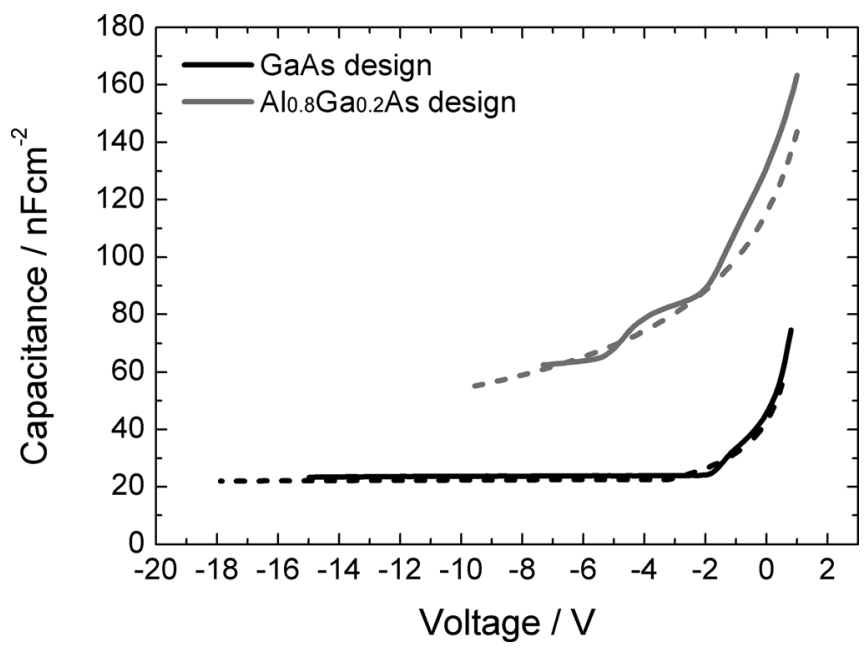

FIG. 4. Shows measured (solid line) and modeled (dashed line) CV data, as used to model multiplication.

Based upon a simple electrostatic model, close agreement was found with the nominal layer thicknesses and doping concentrations, which are given in Figure 1, and the modeled curves are also shown in Figure 4. It should be noted that the features in the experimental data for the AlGaAs design between approximately $-2 \mathrm{~V}$ and $-6 \mathrm{~V}$ result from charge redistribution due to the band offsets around the GaAs/AlGaAs heterointerface, although this is not accounted for in the modeled curve. The associated electric field profiles were next used to model multiplication, using standard integrals. ${ }^{15}$ Parameterized ionization coefficients for GaAs and $\mathrm{Al}_{0.8} \mathrm{Ga}_{0.2} \mathrm{As}$ were taken from Plimmer $^{16}$ and $\mathrm{Ng},{ }^{17}$ respectively. As illustrated in Figure 5, multiplication values could then be found accurately from the experimental data, according to the following procedure. Using the modeled multiplication, data for the primary photocurrent was first calculated for all biases. This was expected to depend on the applied voltage, but to be independent of the multiplication itself. The primary photocurrent was found to be well fitted by an exponential function in each case. This was refined by

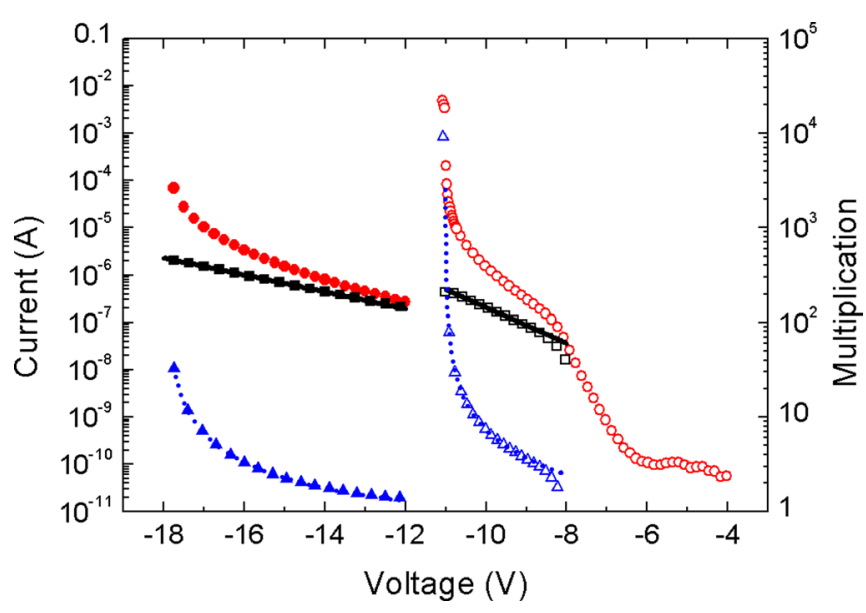

FIG. 5. Shows the calculation of the multiplication, $200 \mu \mathrm{m}$ diameter mesas. Measured photocurrent: GaAs design (O), AlGaAs design (O). Dotted lines: modeled multiplication. Calculated primary photocurrent: GaAs design (ם), AlGaAs Design ( $\square$ ). Solid lines: fitting of primary photocurrent. Calculated multiplication: GaAs design $(\mathbf{\Delta}), \operatorname{AlGaAs}$ design $(\triangle)$. making very small adjustments to the modeled layer profile. These adjustments were cross-checked against the CV data, to ensure good agreement between the two sets of measurements. Finally, the raw photocurrent was divided by the fitted exponentials, giving experimental values for the multiplication.

Excess noise was measured using a calibrated HP 8970B Noise Figure Meter, at frequencies between 20 and $25 \mathrm{MHz}$. The device under test was connected using a $50 \Omega$ impedance-matched cable. Bias was supplied using a Picosecond 5541 A Bias Tee. For analysis, it is common for results to be compared with the theoretical predictions of McIntyre. ${ }^{18}$ Herein, under the local model, the spectral noise density may be written in terms of the total current, the multiplication and the ratio of the ionization coefficients for electrons and holes, $k_{\text {eff }}=\alpha / \beta$. This relation can be reexpressed in the well-known form

$$
F=k_{e f f} M+\left(1-k_{e f f}\right)\left(2-\frac{1}{M}\right),
$$

where $F$ is Excess Noise and $M$ is the multiplication. In the present work, excess noise results were normalized to the above form using a fitting procedure. This step was necessary in order to correct for the electrical coupling of the device with the meter, which results in a constant factor in the noise values measured. The fitting procedure was verified through measurements on $\mathrm{Al}_{0.48} \operatorname{In}_{0.52} \mathrm{As} p$ - $i$ - $n$ devices, where the electrical coupling can also be found experimentally by measurement of the Shot Noise, as detailed elsewhere. ${ }^{19}$ In this analysis, the data in the low $M$ region was disregarded, treating only the data in the near-linear region at high $M$. This choice becomes important when dead space effects are significant, as is the case for the data for the AlGaAs design. To verify that the approach outlined had produced accurate results, duplicate measurements were made on a range of devices, of various diameters, for both samples.

Figure 6 shows data collected for the GaAs design, which lies in the range of $0.2<k_{\text {eff }}<0.4$. Previously reported results for thin GaAs $p-i$ - $n$ diodes are also shown. ${ }^{20}$

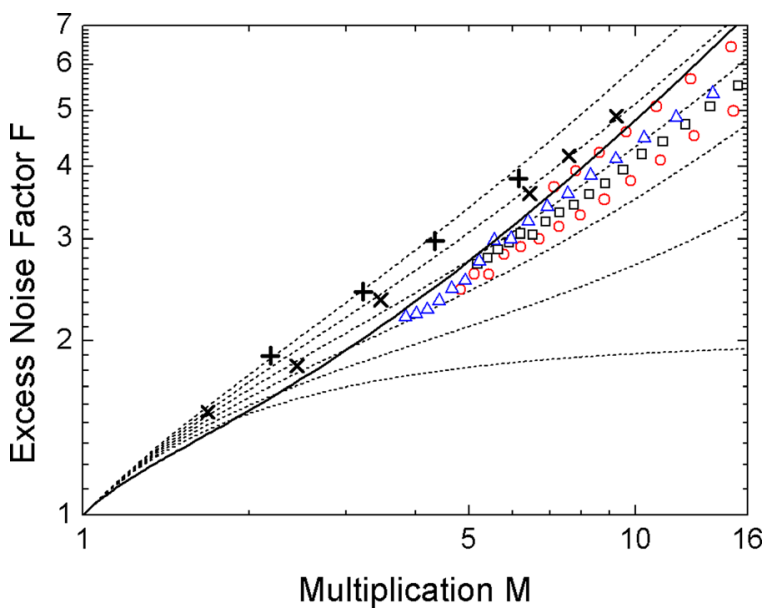

FIG. 6. ( $\square$ ) $200 \mu \mathrm{m}$ diameter device, (○) $100 \mu \mathrm{m}$ diameter devices, $(\triangle)$ $50 \mu \mathrm{m}$ diameter device, Solid line: RPL Simulation, $(\times)$ GaAs $p-i-n$ with $0.28 \mu \mathrm{m}$ intrinsic width [Li, 1998], (+) GaAs $p-i-n$ with $0.49 \mu \mathrm{m}$ intrinsic width [Li, 1998], Dashed lines: local model curves of Eq. (1), from $\mathrm{k}=0$ to $\mathrm{k}=0.5$, in steps of 0.1 . 


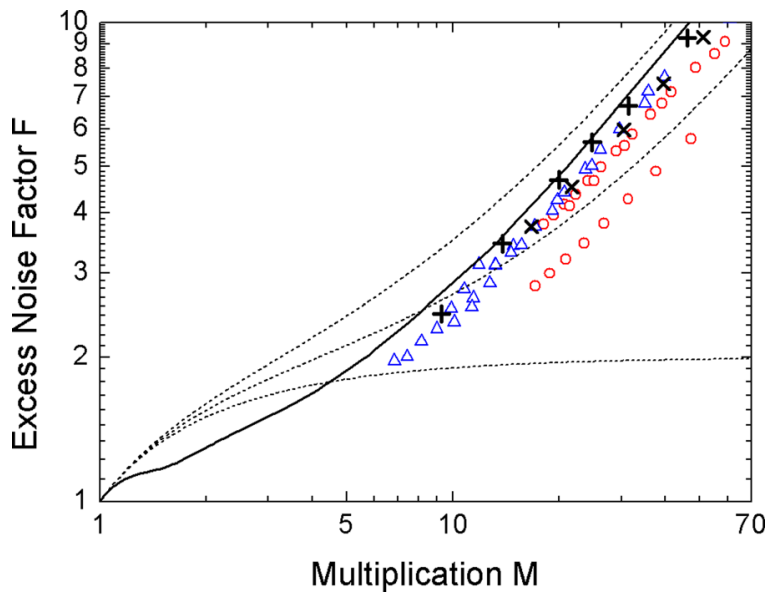

FIG. 7. (○) $100 \mu \mathrm{m}$ diameter devices, $(\triangle) 50 \mu \mathrm{m}$ diameter devices, Solid line: RPL Simulation, $(\times) \mathrm{Al}_{0.8} \mathrm{GaAs} p-i-n$ with $0.03 \mu \mathrm{m}$ intrinsic width [ $\left.\mathrm{Ng}, 2002\right]$, (+) $\mathrm{Al}_{0.8} \mathrm{GaAs} p-i$ - $n$ with $0.10 \mu \mathrm{m}$ intrinsic width [Ng, 2002], dashed lines are the local model curves of Eq. (1), from $\mathrm{k}=0$ to $\mathrm{k}=0.2$ in steps of 0.1 .

The lower value of $k_{\text {eff }}$ apparent in our devices may be explained based on their higher doping concentration in the multiplication region, so that they approach a $p-n$ design. This results in a localization of the high electric field and, therefore, the impact ionization, at the type junction. By decreasing the length over which ionization events are concentrated, the level of disorder and, therefore, the values of $k_{\text {eff }}$ measured are reduced in turn. A random-path-length (RPL) model was used to investigate this effect; results from the model are shown in Figure 6. The modeled excess noise characteristic can be seen to agree closely with the experimental data. Ionization threshold energies of $2.3 \mathrm{eV}$ for electrons and $2.1 \mathrm{eV}$ for holes were used. ${ }^{20}$ The RPL model takes into account a variable field profile, but is otherwise similar to the work of DS Ong. ${ }^{21}$

Data collected for the AlGaAs design is shown in Figure 7. This time, a significant deviation from the form of the local model curves can be seen in the region $M<20$. This effect is attributed to the increased fraction of dead-space in the narrow structure. This is corroborated by an RPL model curve, for which ionization threshold energies of $3.75 \mathrm{eV}$ were used, for both electrons and holes. Except for one outlier, the data was contained in the range of $0.1<k_{\text {eff }}<0.2$. This closely agrees with published data for thin $\mathrm{Al}_{0.8} \mathrm{Ga}_{0.2}$ As diodes. ${ }^{22}$

At present, only low quantum efficiencies could be obtained in both devices. This problem is believed to be linked with the band offsets at the GaSb heterojunction and, in the AlGaAs design, additionally with the band offsets at the $\mathrm{Al}_{0.8} \mathrm{Ga}_{0.2} \mathrm{As} / \mathrm{GaAs}$ heterojunction. The effects of the $\mathrm{GaSb}$ band offset could be mitigated using a doping-interface-dipole approach. $^{23}$ Thicker absorption regions and anti-reflective coatings could also be used. Larger photocurrents would also allow correction for the electrical coupling using a shot noise measurement, as discussed above.

Two APD structures were demonstrated, each based on a lattice-mismatched GaSb absorber region grown using an IMF array. The first was based on a GaAs design, representing the simplest case. The second device used an AlGaAs design, allowing for lower noise and dark currents and a thinner multiplication region. Excess noise results were compared with data from similar structures, without long- wavelength absorber regions or mismatched epitaxial interfaces. Agreement was found, indicating an absence of ionization effects associated with the $\mathrm{GaSb}$ regions or the interface, which could affect device performance. Comparisons were also made with curves generated using an RPL model, highlighting a dependence on the field profile and dead-space effects. It is envisaged that further designs, based on longer wavelength absorber materials which are lattice-matched to GaSb, could also be developed.

The authors wish to thank the UK Engineering and Physical Sciences Research Council for the studentship provided to A. P. Craig (Grant No. EP/P505585/1) and acknowledge the support of the US Department of Defense (DoD) for the Science, Mathematics, and Research for Transformation Scholarship provided to C. J. Reyner. In addition, we wish to acknowledge the support of the DoD for NSSEFF Grant N00244-09-1-0091 and the Royal Academy of Engineering Fellowship awarded to A. R. J. Marshall (Grant number EP/H043993/1).

${ }^{1}$ I. M. Baker, S. S. Duncan, and J. W. Copley, Proc. SPIE 5406, 133 (2004).

${ }^{2}$ A. Krier and W. Suleiman, Appl. Phys. Lett. 89, 083512 (2006).

${ }^{3}$ A. R. J. Marshall, C. H. Tan, M. J. Steer, and J. P. R. David, Appl. Phys. Lett. 93, 111107 (2008); IEEE Photonics Technol. Lett 21(13), 866 (2009).

${ }^{4}$ S. Mallick, K. Banerjee, S. Ghosha, E. Plis, J. B. Rodriguez, S. Krishna, and C. Grein, Appl. Phys. Lett. 91, 241111 (2007).

${ }^{5}$ Y. L. Goh, D. S. G. Ong, S. Zhang, J. S. Ng, C. H. Tan, and J. P. R. David, IEEE Lasers and Electro-Optics Society, LEOS Annual Meeting Conference Proceedings 2009, 4-8 October 2009 (IEEE, 2009), pp. 293-294.

${ }^{6}$ E. K. Duerr, M. J. Manfra, M. A. Diagne, R. J. Bailey, J. P. Donnelly, M. K. Connors, and G. W. Turner, Appl. Phys. Lett. 91, 231115 (2007).

${ }^{7}$ O. V. Sulima, M. G. Mauk, Z. A. Shellenbarger, J. A. Cox, J. V. Li, P. E. Sims, S. Datta, and S. B. Rafol, in IEE Proc.: Optoelectron. 151(1), 1-5 (2004).

${ }^{8}$ S. H. Huang, G. Balakrishnan, A. Khoshakhlagh, A. Jallipalli, L. R. Dawson, and D. L. Huffaker, Appl. Phys. Lett. 88, 131911 (2006).

${ }^{9}$ S. Huang, G. Balakrishnan, and D. L. Huffaker, J. Appl. Phys. 105, 103104 (2009).

${ }^{10}$ M. Mehta, G. Balakrishnan, S. Huang, A. Khoshakhlagh, A. Jallipalli, P. Patel, M. N. Kutty, L. R. Dawson, and D. L. Huffaker, Appl. Phys. Lett. 89, 211110 (2006).

${ }^{11}$ J. B. Rodriguez, L. Cerutti, and E. Tournié, Appl. Phys. Lett. 94, 023506 (2009).

${ }^{12}$ K. C. Nunna, S. L. Tan, C. J. Reyner, A. R. J. Marshall, B. Liang, A. Jallipalli, J. P. R. David, and D. L. Huffaker, IEEE Photonics Technol. Lett. 24, 218 (2012).

${ }^{13}$ A. Jallipalli, K. Nunna, M. N. Kutty, G. Balakrishnan, G. B. Lush, L. R. Dawson, and D. L. Huffaker, Appl. Phys. Lett. 95, 072109 (2009).

${ }^{14}$ S. Sridaran, A. Chavan, and P. S. Dutta, J. Cryst. Growth 310, 1590 (2008).

${ }^{15}$ G. E. Stillman and C. M. Wolfe, "Avalanche photodiodes," in Infrared Detectors II, Semiconductors and Semimetals Vol 12, edited by R. K. Willardson and A. C. Beer (Academic Press, New York, 1977), pp. 291-393.

${ }^{16}$ S. A. Plimmer, J. P. R. David, and D. S. Ong, IEEE Trans. Electron Devices 47, 1080 (2000).

${ }^{17}$ B. K. Ng, J. P. R. David, S. A. Plimmer, M. Hopkinson, R. C. Tozer, and G. J. Rees, Appl. Phys. Lett 77(26), 4374 (2000).

${ }^{18}$ R. J. McIntyre, IEEE Trans. Electron Devices 13(1), 164 (1966).

${ }^{19}$ J. C. Campbell, S. Chandraskhar, W. T. Tsang, G. J. Qua, and B. C. Johnson, J. Lightwave Technol. 7(3), 473 (1989).

${ }^{20}$ K. F. Li, D. S. Ong, J. P. R. David, G. J. Rees, R. C. Tozer, P. N. Robson, and R. Grey, IEEE Trans. Electron. Devices 45(10), 2102 (1998).

${ }^{21}$ D. S. Ong, K. F. Li, G. J. Rees, J. P. R. David, and P. N. Robson, J. Appl. Phys. 83(6), 3426 (1998).

${ }^{22}$ B. K. Ng, J. P. R. David, R. C. Tozer, M. Hopkinson, G. Hill, and G. J. Rees, IEEE Photonics Technol. Lett. 14(4), 522 (2002).

${ }^{23}$ F. Capasso, A. Y. Cho, K. Mohammed, and P. W. Foy, Appl. Phys. Lett. 46(7), 664 (1985). 